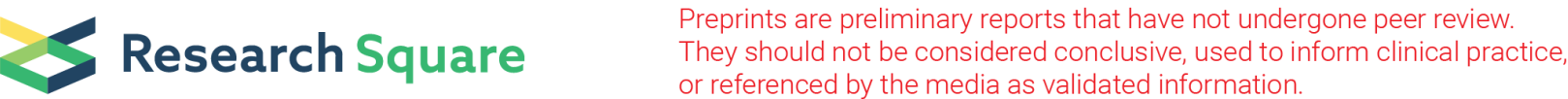

\section{G-CSF-mobilized Peripheral Blood Mononuclear Cells Combined with Platelet-rich Plasma Restored the Ovarian Function of aged rats via angiogenesis and glycolysis modulation}

\section{Lifang Yuan}

the First Affiliated Hospital of Guangxi Medical University

\section{Weiyu Huang}

the First Affiliated Hospital of Guangxi Medical University

Yin Bi

the First Affiliated Hospital of Guangxi Medical University

\section{Saiqiong Qin}

the Fourth Affiliated Hospital of Guangxi Medical University

\section{Xi Wang}

the First Affiliated Hospital of Guangxi Medical University

Ting Li

the First Affiliated Hospital of Guangxi Medical University

\section{Peiru Wei}

the First Affiliated Hospital of Guangxi Medical University

Jiebing Du

Guangxi Maternal and Child Healthcare Hospital

\section{Ling Zhao}

the First Affiliated Hospital of Guangxi Medical University

Bo Liu

the First Affiliated Hospital of Guangxi Medical University

Yihua Yang ( $\nabla$ workyyh@163.com )

the First Affiliated Hospital of Guangxi Medical University

\section{Research Article}

Keywords: Natural ovarian aging (NOA), PBMCs, PRP, Angiogenesis, Glycolytic

Posted Date: October 3rd, 2022

DOI: https://doi.org/10.21203/rs.3.rs-1182718/v2 
License: (c) (i) This work is licensed under a Creative Commons Attribution 4.0 International License. Read Full License 


\section{Abstract \\ Background}

Regenerative medicine with peripheral blood mononuclear cells (PBMCs) transplantation sheds light on the issue of premature ovarian insufficiency (POI). However, the efficiency of PBMCs treatment in patients with natural ovarian aging (NOA) remain unclear.

\section{Methods}

13-month-old female Sprague-Dawley (SD) rats were used to verify NOA model. Seventy-two NOA rats were randomly divided into three groups: the NOA control group, PBMCs group, and PBMCs + PRP group. PBMCs and PRP were transplanted by intraovarian injection. The effects of ovarian function and fertility ability were measured after transplantation.

\section{Results}

Transplantation of PBMCs could restoration of the normal estrus cycle, consistent with recovery of serum sex hormone levels, increased follicle numbers at all stages, and restoration of fertility by facilitating pregnancy and live birth. Moreover, when combined with PRP injection, these effects were more significant. The male-specific SRY gene was detected in the ovary at all four time points, suggesting PBMCs continuously survived and functioned in NOA rats. In addition, after PBMCs treatment, the expression of the angiogenesis-related and glycolysis-related markers in ovarian were upregulated, which indicated that these effects were associated with angiogenesis and glycolysis.

\section{Conclusions}

PBMCs transplantation restore the ovarian functions and fertility of NOA rats by increasing ovarian vascularization, folliculogenesis and glycolysis, and PRP could enhance the efficiency.

\section{Introduction}

Aging is a universal natural destiny in organisms and a normal process that occurs when the body grows and matures with age. Aging is also an irreversible problem that the body will eventually face and cannot avoid(Ren \& Zhang, 2018). Aging is accompanied by changes in behavior, neuroendocrine function, immune regulation, and other physiological functions. With the aggravation of aging, the body will gradually lose its ability to adapt to the environment and become vulnerable(Beard et al., 2016). Significantly, reproductive function is more vulnerable to aging. In female reproductive organs, ovaries play an essential role in maintaining the stability of endocrine and reproductive functions. Aging in ovaries occurs much earlier than in other body tissues and organs(Tatone et al., 2008). 
Natural ovarian aging (NOA), also known as ovarian physiologic aging, is a worldwide reproductive health problem(White et al., 2012). It is a generalized, multifaceted, complex, and progressive degradation process affected by genetics, environmental conditions, and lifestyles with age(Beard et al., 2016). As the quantity and quality of ovaries decline with aging, women will face fertility reduction in a lifespan(Broekmans, Soules, \& Fauser, 2009). NOA interferes with fertility and causes menopausal symptoms as well as severe psychological sequelae. Moreover, NOA is a devastating medical condition with minimal treatment options. As the population of older women is rising globally and an increasing number of women are willing to delay marriage and childbearing, the demand for efficient strategies that restore ovarian functions in NOA patients is increasing.

Currently, hormone replacement therapy (HRT), ovarian tissue cryopreservation, stem cell transplantation, and artificial ovaries have been applied to patients with ovarian dysfunction(Dadashzadeh, Moghassemi, Shavandi, \& Amorim, 2021; Grosbois, Devos, \& Demeestere, 2020; Khaleghi et al., 2021; Seok et al., 2020). However, the efficiency of these therapies lacks evidence. Thus, there is an urgent need to develop novel, effective passageways for NOA treatment.

Recently, peripheral blood mononuclear cells (PBMCs) therapy has emerged as a novel option for tissue and organ regeneration and angiogenesis(Kang et al., 2012; Koh et al., 2018; Wan Jamaludin et al., 2018). It has been reported that intravenous infusion of nucleated peripheral blood cells restores fertility in chemotherapy-induced premature ovarian failure (POF) mice(El Andaloussi, Igboeli, Amer, \& Al-Hendy, 2018). However, the efficacy of PBMCs in NOA has not yet been investigated. On the other hand, plateletrich plasma (PRP), derived from peripheral blood, is another well-known factor that stimulates and accelerates tissue regeneration, skeletal muscle repair, wound healing, scar repair, skin regeneration, and alopecia(Alves \& Grimalt, 2018; Aydin et al., 2018). It was reported that PRP promotes the development of presinusoidal follicles in POF rats(Ahmadian et al., 2020). It is encouraging that PRP could enhance the proliferation and differentiation of transplanted cells. Therefore, PRP often combines with PBMCs in transplantation(Sassoli et al., 2018).

Stem cell transplantation is a promising treatment. Previous studies suggest that the fundamental mechanism by which stem cell therapy works is to promote angiogenesis, but other potential mechanisms are still unknown(Aziz, Yusop, \& Ahmad, 2020). Studies have shown that glycolysis is very important for folliculogenesis and that the level of glycolysis changes during oocyte aging(Fontana, Martínková, Petr, Žalmanová, \& Trnka, 2020). Therefore, whether the regulation of glycolysis in the ovary is also regulated by PBMCs transplantation raised our interest.

In our previous study, we reported that PBMCs were efficient in treating cyclophosphamide (CTX)-induced POI rats and that combination with PRP could improve this effect(Huang et al., 2019). In addition, a recent study found that compared with intraperitoneal injection and tail intravenous injection, intraovarian injection was a more conspicuous result effect for purified MSC transplantation in a mouse model(Wang et al., 2021). Therefore, in this study, we will use PBMCs combined with PRP to recover 
ovarian function in the NOA rat model via intraovarian injection. Our study will also investigate the possible link between granulosa glycolysis and ovarian function in NOA rats.

\section{Materials And Methods \\ 4.1. Study designing}

The animal study is divided into two parts (Fig. S1). In Part I, 36 NOA rats were divided into the NOA control group, PBMCs, and PBMCs + PRP transplantation groups. The day of transplantation was recorded as D0, and the ovarian function of the three groups was evaluated at D5, D10, D15, and D20. The observation indices included the estrous cycle, serum sex hormone levels (AMH, E2, and FSH), follicle count, and so on. In part II, based on the data analysis from Part I, which is that the recovery of ovarian function in the transplantation groups reached a peak on D10 after transplantation, the fertility status of the other 36 NOA rats was evaluated by mating trials starting on D10. More details can be found in the specific method in the fertility examination section.

\subsection{Experimental animal}

Eighty 13-month-old female SD rats were used to verify the establishment of the NOA rat model, and seventy-two NOA rats were randomly divided into three groups: the NOA control group, PBMCs group, and PBMCs combined with platelet-rich plasma (PRP) group (PBMCs + PRP group). We also used six 12-weekold SD female rats with regular estrus cycles as young controls. Moreover, twenty-four 5-week-old male SD rats weighing between 90 and $100 \mathrm{~g}$ were used to isolate PBMCs and PRP. Rats were purchased from the Experimental Animal Center of Guangxi Medical University, cultivated at a temperature of $28 \pm 2^{\circ} \mathrm{C}$, and had a 12-hour light/dark cycle. All animal procedures were conducted by the Ethics Committee of the experimental Animal Center of Guangxi Medical University for the care and use of laboratory animals (NO.202003002).

\subsection{Isolation of PBMCs}

Sixteen adolescent male rats were subcutaneously injected daily with G-CSF (Qilu Pharmaceutical Co, Ltd, China) at a dose of $100 \mu \mathrm{g} / \mathrm{kg} /$ day for 5 days. Blood samples were collected $12 \mathrm{~h}$ after the last injection from the abdominal aortas of anesthetized rats. PBMCs were harvested by density gradient centrifugation (Eppendorf, Hamburg, Germany) with FICOLL PLUS 1.083 (Solarbio, China) according to the manufacturer's instructions. PBMCs were washed twice with PBS followed by centrifugation at $250 \times$ $\mathrm{g}$ for $10 \mathrm{~min}$ at $4^{\circ} \mathrm{C}$ and then resuspended in PBS. After counting, the PBMCs were stored at $4^{\circ} \mathrm{C}$ for transplantation use but for no more than four hours.

\subsection{Isolation of PRP}

Blood from eight adolescent male rats without G-CSF mobilization was collected from the abdominal aorta and transferred into test tubes containing 3.2\% sodium citrate (L14068, ShoeBio, China) at a blood/citrate rate of $9 / 1$. After centrifugation at $250 \times \mathrm{g}$ for $10 \mathrm{~min}$, the supernatant was transferred to a 
new tube and centrifuged at $1000 \times \mathrm{g}$ for $10 \mathrm{~min}$. Removing the top layer and the $0.5 \mathrm{~mL}$ leftover was considered PRP and, after counting, stored at 4॰C for transplantation use but no more than four hours.

\subsection{Estrus cycle observation and NOA rat identify}

Cotton swabs took vaginal secretions of each rat at 10:00 am every day to make a smear. These smears were dried at room temperature and stained with Swiss dye (G1040, Solarbio) for 1 min, and cell types and their proportions were quantified under an optical microscope (Bx53F, Olympus) to estimate the different phases of estrus. Only those who showed abnormal estrus (over 5 days did not appear estrous cycles) by vaginal smears were defined as NOA rats.

\subsection{Intraovarian injection of PBMCs and PBMCs combined with PRP}

Rats were anesthetized and underwent surgery under a sterile environment. Bilateral ovaries were exposed, and the surrounding adipose tissues were carefully stretched to stabilize the ovary. The concentrated cell suspension was extracted by a microsyringe and injected into each ovary. Notably, the PBMCs group received an intraovarian injection of $4 \times 10^{6}$ PBMCs per ovary, and the PBMCs + PRP group received an intraovarian injection of $4 \times 10^{6} \mathrm{PBMCs}$ combined with $4 \times 10^{7} \mathrm{PRP}$ platelets on each side. A local upheaval was invisible after injection. The needle stayed in the injection site for $30 \mathrm{~s}$ followed by gentle pull-out. A gentle and soft operation was required during the procedure to avoid injury to ovarian blood vessels. Post-injection observation was required to avoid bleeding and the spillover of transplantation suspension. The day of cell transplantation refers to Day 0. After that, the Sry gene was used to track the transplanted PBMCs in the ovaries of the transplanted group.

\subsection{Fertility examination}

After analyzing the curative effect of cell transplantation in NOA rats, we chose the peak of AMH hormone level curve D10, which means the peak of ovarian function recovery for the fertility experiment. On the 10th day of cell transplantation, we began to mate overnight with female rats and sexually mature male rats at 2:1, and the vaginal suppository was examined at 8 am every morning to determine whether the mating was successful. If the vaginal suppository was positive, it was defined as the first day of pregnancy, marked D1. Five rats in each group were anesthetized on the 9th-12th day of pregnancy to check the number of embryos. Afterward, the remaining successfully mated rats will be caged individually until parturition, and the litter number per rat will be recorded.

\subsection{Serum sex hormone detection}

Blood was collected when rats were sampled. The blood samples were left at room temperature for 1 hour. The serum was collected by centrifuging blood at $3000 \mathrm{rpm}$ for 10 minutes at $4^{\circ} \mathrm{C}$ and then stored at $-80^{\circ} \mathrm{C}$ for further use. Serum concentrations of FSH (CEA830Ra, Cloud-Clone Corp, Wuhan, China), E2 (CEA461Ge, Cloud-Clone Corp), and AMH (CEA228Ra, Cloud-Clone Corp) were measured by ELISA according to the manufacturer's instructions. 


\subsection{Sampling and histopathological studies}

On the 5 th, 10th, 15th, and 20th days after transplantation, the ovaries of each group were collected $(\mathrm{n}=$ 2). Rats were anesthetized by intraperitoneal injection of $10 \%$ chloral hydrate prior, and ovaries were removed and rinsed in phosphate-buffered saline (PBS) solution. Then, one side of the ovary was stored in liquid nitrogen until use. The other side was fixed in neutral-buffered formalin overnight and embedded in paraffin, which was further serially sectioned at $5 \mu \mathrm{m}$ and stained with hematoxylin-eosin for histology. Imaging was performed by using an optical microscopic photography system (Hamamatsu, Japan), and images were processed using NDP.VIEW. 2.6.17 software (Hamamatsu Photonics).

\subsection{Immunohistochemical (IHC) staining and score analysis}

Ovarian tissue sections of each group at Day 10 were selected for IHC. Briefly, after dewaxing and rehydrating the tissue section, high-temperature antigen unmasking, as recommended by each supplier, was performed, and sections were incubated overnight at $4^{\circ} \mathrm{C}$ with the following antibodies: $\mathrm{AMH}$ (1:500, ab272221, Abcam, Inc., UK), FSHR (1:200, NBP2-36489, Novus, CHN), CD31 (1:600, ab182981, Abcam, Inc., UK), VEGF (1:500, ab231260, Abcam, Inc., UK), a-SMA (1:500, ab124964, Abcam, Inc., UK), SIRT1 (1:600, ab189494, Abcam, Inc., UK) and LDH-A (1:500, ab52488, Abcam, Inc., UK).

The intensity of $\mathrm{IHC}$ staining was calculated according to the $\mathrm{H}$-score equation: $\mathrm{H}$ score $\sum \mathrm{Pi}(\mathrm{i}+1)$, where $\mathrm{i}$ is the intensity of staining ( $0=$ negative; $1=$ weak; $2=$ moderate; and $3=$ strong $)$ and $\mathrm{Pi}$ is the percentage of cells stained at each intensity $(0-100 \%)$. The $\mathrm{H}$-scores were measured in stromal cells and granulosa cells separately. Sections were scored by two observers independently.

\subsection{Histomorphometric analysis of the vessels}

To assess the angiogenic potential after transplantation with PBMCs or combined with PRP in ovarian tissue. Vascular density was examined by IHC staining. We performed IHC staining of CD31 and anti-aSMA antibodies. In this study, counting of the blood vessels was carried out at a 200 -fold field, and five high-point fields from three different sections were chosen for each sample.

\subsection{Immunofluorescence(IF) staining}

The protein expression of CD31 and a-SMA was detected by immunofluorescence staining. Briefly, after being deparaffinized, rehydrated, and washed in $0.01 \mathrm{M} \mathrm{PBS}(\mathrm{pH}=7.2)$, sections were incubated with $3 \%$ $\mathrm{H} 2 \mathrm{O} 2$ for 10 min at room temperature to block endogenous peroxidase, subjected to high-pressure antigen recovery sequentially in $0.01 \mathrm{M}$ citrate buffer $(\mathrm{pH}=6.0)$ for $5 \mathrm{~min}$, washed three times in PBS, and incubated with blocking solution (5\% goat serum and $0.1 \%$ BSA in PBS) for $2 \mathrm{~h}$ at room temperature and then with diluted primary antibodies overnight at $4^{\circ} \mathrm{C}$. The following primary antibodies were used: CD31 (1:600, ab182981, Abcam, Inc., UK) and a-SMA (1:500, ab124964, Abcam, Inc., UK). After washing with PBS, sections were incubated with appropriate secondary antibodies for one hour, Alexa Fluor® 594 
rabbit anti-goat IgG or Fluor ${ }^{\circledR} 488$ goat anti-rat IgG. Nuclei were stained with $0.5 \mu \mathrm{g} / \mathrm{ml}$ DAPI. Slides were stored in the dark at $4^{\circ} \mathrm{C}$ prior to microscopy (Bx53F, Olympus, Japan). Images were taken from five high point fields $(\times 200)$, and the positive area was counted on Image-Pro Plus 6.0.

\subsection{Quantitative real-time PCR (qPCR)}

Total RNA was extracted with TRIzol reagent (Thermo Fisher Science, Germany) combined with chloroform extraction and ethanol precipitation. cDNA was synthesized using the Revert Aid First Strand cDNA Synthesis Kit (K1622, Thermo Fisher Science). Real-time PCR analyses for the gene expression level were performed on the Applied Biosystems 7500 Real-time PCR System (Applied Biosystems, Foster City, CA, USA). GAPDH was used as a reference control, and gene expression levels were calculated using the comparative $\mathrm{Ct}$ method. PBMCs can be tracked by continuous Sry gene expression. The primers were used to detect Sry gene expression in female recipient rats(Ahmed et al., 2016). The PCR product was separated by $0.2 \%$ agarose gel electrophoresis, and the results were processed by Image Lab version 4.01 software. See Table 1 for the gene sequence.

Table 1

Sequences of PCR primers and length of products.

\begin{tabular}{|c|c|c|c|}
\hline Gene & Sequence of primer $\left(5^{\prime}-3^{\prime}\right)$ & Gene Bank No. & Length of product(bp) \\
\hline \multirow[t]{2}{*}{ GAPDH } & F: CCTCAAGATTGTCAGCAATG & NM-017008.4 & 134 \\
\hline & R: CAGTCTTCTGAGTGGCAGTG & & \\
\hline \multirow[t]{2}{*}{ SRY } & F:CATCGAAGGGTTAAAGTGCCA & NM-012772.1 & 104 \\
\hline & R:ATAGTGTGTAGGTTGTTGTCC & & \\
\hline \multirow[t]{2}{*}{ SIRT1 } & F:AGATACCTTGGAGCAGGTTGC & NM-001372090.1 & 117 \\
\hline & R:TCTCCACGAACAGCTTCACAA & & \\
\hline \multirow[t]{2}{*}{ LDH-A } & F: GGTTGACAGTGCATACGAAT & NM-017025.1 & 106 \\
\hline & R: CCGCCTAAGGTTCTTCATTA & & \\
\hline \multirow[t]{2}{*}{ HK } & F: AGAGGCTACGGACAGAGATG & NM-012734.1 & 121 \\
\hline & R: AGGAAGTCACCGTGTTCAGT & & \\
\hline \multirow[t]{2}{*}{ PFK } & F: GGCGTGTGTTCATTGTAGAG & NM-017008.4 & 125 \\
\hline & R: CTTCAAGTCGTGGATGTTGA & & \\
\hline \multirow[t]{2}{*}{ PKM } & F: ACATCCTGTGGCTGGACTAT & NM-053297.2 & 130 \\
\hline & R: TCCACTTCTGTCACCAGGTA & & \\
\hline
\end{tabular}

\subsection{Statistical analysis}


All experiments were repeated at least three times. The statistical analyses were performed using SPSS 20.0. One-way ANOVA was used to determine significant differences among the three groups. All data are presented as the mean \pm SD. All statistical charts were created on GraphPad Prism 7 (San Diego). $* \mathrm{P}<$ $0.05, * * \mathrm{P}<0.01, * * * \mathrm{P}<0.001$. A $P$ value less than 0.05 was considered to be statistically significant.

\section{Result}

\subsection{Verification of NOA rat model}

To verify the NOA model, we assessed and compared several specific physiological indices between 13month-old SD rats and young control rats (Fig. 1). Compared with NOA control ovaries, the ovaries from the young control group were larger, and the ovarian cortex medulla was clear. The follicles at all stages were active, arranged regularly, and developed normally. In contrast, the NOA control rats had severe ovarian atrophy and decreased volumes (Fig. 1A). In addition, the ovaries of NOA control rats showed disordered cortical and medullary structures, a portion of the zona pellucida was abnormal, and the number of follicles at all levels was significantly reduced (Fig. 1B, 1C). Compared with young control rats, the estrous cycle of NOA control rats was irregular, and the estrous cycle could not be observed in 5 days (Fig. 1D). The ovarian/body weight ratio of NOA control rats was significantly lower (Fig. 1E). More importantly, the levels of AMH and E2 significantly decreased in NOA control rats, while the levels of FSH obviously increased (Fig. 1F, 1G, 1H). At the same time, the number of follicles of NOA control rats at all stages was significantly reduced compared to that in young control rats (Fig. 1I). These results suggested that the NOA rat model was suitable for our subsequent study.

\subsection{Tracking of transplanted PBMCs}

To ensure that the PBMCs were transplanted and survived, the male rat specific gene Sry expression in ovaries was analyzed. The PCR results showed that Sry was detected in the ovarian tissues of PBMCs and PBMCs + PRP group rats at all four time points after PBMCs or PBMCs + PRP transplantation. Interestingly, the Sry gene was still detected until the 15th day when combined with PRP, which was longer than PBMC transplantation alone, indicating that PRP contributes to prolonging PBMC survival (Fig. 2A).

\subsection{Ovarian function was restored by PBMCs and PBMCs + PRP in an NOA rat model}

\section{Ameliorates estrus cycle disorder}

Compared with the NOA control group, the rats in the PBMCs and PBMCs + PRP groups showed recovery of the estrous cycle, which was similar to the route of the young control group. Meanwhile, the estrous cycle of NOA control group rats remained irregular throughout the entire experiment, which suggested 
that PBMCs and PBMCs + PRP transplantation facilitated the amelioration of sexual cycle disorder (Fig. 2B).

\section{Regulates the sex hormone expression}

The serum sex hormones of all groups were examined on the 5th, 10th, 15th, and 20th days after transplantation. The expression of $\mathrm{AMH}$ and $\mathrm{E} 2$ in the rats in the PBMCs and PBMCs + PRP groups increased gradually until reaching a peak at Day 10 and then decreased (Fig. 2C, 2D). The peak levels were closed to the young control. Meanwhile, the expression of FSH was contradictory, which was decreased before the day of the 15th and then increased (Fig. 2E). This suggested that PBMCs and PBMCs + PRP transplantation regulates aberrant hormone expression in NOA rats, and the effect of combined use is more effective.

\section{Increasing the number of follicles}

We counted the number of follicles at all stages of the three groups of rats on the 5th, 10th, 15th, and 20th days after transplantation. The results showed that the number of follicles at all levels in the PBMCs group was significantly increased compared to that in the NOA control group. The number of primordial and primary follicles increased significantly on the 15th day, and the number of secondary and antral follicles increased significantly on the 10th day. The increase in follicles was more significant in the PBMCs + PRP group (Fig. 2F, 2G, 2H, 2I). These results indicate that PBMC transplantation may restore ovarian function in NOA rats to some extent and that combination with PRP was more effective.

\section{Facilitating pregnancy and live birth}

To explore whether PBMCs and PBMCs + PRP transplantation improve the fertility of NOA rats, the embryo-implantation efficiency and the pregnancy rate were examined in each group. As shown in Fig. 3G, the time to pregnancy in the NOA control group was significantly longer than that in the PBMCs and PBMCs + PRP groups ( $<0.05)$, which means that the NOA control rats after cell transplant treatment were more likely to become pregnant. As shown in Fig. $3 \mathrm{H}$ and $3 \mathrm{l}$, the efficiency of embryo implantation and live birth rates in the NOA control group were significantly lower than those in the PBMCs and PBMCs + PRP groups. As expected, the combined use of PBMCs and PRP increased the implantation rates and live birth rates more effectively. In summary, the above data suggested that PBMCs + PRP transplantation can restore the fertility of NOA rats.

\subsection{PBMCs and PBMCs + PRP significantly increase the expression of angiogenesis- and folliculogenesis-related markers}

To explore the potential mechanisms of ovarian function restoration by PBMCs + PRP transplantation, we assessed ovarian folliculogenesis and angiogenesis-related markers at Day 10 after transplantation. The expression of $\mathrm{AMH}$, mainly localized to primary follicles, sinus follicles, and the corpus luteum, was 
relatively high in the PBMCs and PBMCs + PRP groups compared to the NOA control group (Fig. 4A). FSHR was mainly observed in sinus follicles, preovulation follicles, and the corpus luteum in the transplantation groups except for the NOA control group, and expression was more robust in the PBMCs + PRP group but weaker in the PBMCs group (Fig. 4B). The expression of VEGF in the interstitium and corpus luteum was significantly higher in the PBMCs and PBMCs + PRP groups than in the NOA control group (Fig. 4C). Significantly increased numbers of CD31-positive and a-SMA-positive cells were observed in the vascular wall around each follicle and in numerous capillaries uniformly spread in the cortex after transplantation (Fig. 4D, 4E).

To evaluate the effect of angiogenesis in NOA more obviously, we detected the protein expression of CD31 and a-SMA in each group by IF on Day 10 (Fig. 5). The periendothelial cell area and endothelial cell area in the ovary were evaluated by counting CD31-positive cells and a-SMA-positive cells per high-power field, respectively. The results showed that the percentages of CD31- and a-SMA-positive areas were significantly decreased in the NOA group compared to the PBMCs and PBMCs + PRP groups (Fig. 5A, 5B). PBMCs combined with PRP more effectively upregulated ovarian angiogenesis. The injection of PBMCs and PBMCs + PRP increased the vascular density on Day 10 after treatment. The ovarian blood vessels were counted by immunofluorescence staining of CD31 and a-SMA. The data showed that the application of PBMCs alone or in combination with PRP in NOA rats could enhance the blood vessel density and blood supply in ovarian tissue (Fig. 5C).

\subsection{Effects of cell transplantation treatment on ovarian glycolysis-related rate-limiting enzymes and SIRT1 expression}

The expression of lactate dehydrogenase A (LDH-A) and sirtuin 1 (SIRT1) was detected by immunohistochemistry. SIRT1 protein was expressed in follicular granular cells, including ovarian cumulus. Compared with the young control group, the number of SIRT1-positive cells in the NOA control group was significantly decreased. After transplantation, the protein expression of SIRT1 was significantly increased (Fig. 6A). The same effect was also consistent in LDH-A (Fig. 6B). Furthermore, the expression of SIRT1, LDH-A, and glycolysis-related rate-limiting enzymes in the ovary was analyzed by qRT-PCR. The mRNA expression of hexokinase (HK), phosphofructokinase (PFK), pyruvate kinase isozyme M2 (PKM2), SIRT1, and LDH-A in ovaries from the NOA control group was lower than that in ovaries from the young control group and was increased after PBMCs and PBMCs + PRP treatment (Fig. 6C, 6D, 6E). These results suggested that PBMCs treatment may be associated with glycolysis enhancement.

\subsection{Possible signaling pathways in the study}

The schematic diagram illustrates the possible mechanisms responsible for the restorative effects of PBMCs + PRP-based therapy in Supplementary FigS2. The paracrine proteome of VEGF functions in the 
ovarian microenvironment of a natural ovarian aging rat model by promoting angiogenesis and glycolytic pathway activity. The paracrine proteome signaling function in the ovarian microenvironment with resistance to natural ovarian aging may mainly be upregulated by angiogenesis-, glycolysis-, and folliculogenesis-related genes. As a consequence, the ovarian function of NOA rats recovered.

\section{Discussion}

Human follicle development strictly depends on angiogenesis, which enables the follicle to receive the necessary supply of nutrients and oxygen and facilitates the release of steroids(Q. Xie, Cheng, Chen, Lobe, \& Liu, 2017). The current knowledge and understanding of female reproductive aging is limited. Previous studies have shown that decreased ovarian function is a consequence of excessive dormant primordial follicle activation(Kalich-Philosoph et al., 2013), massive granulosa cell apoptosis(Spears et al., 2019), persistent vascular damage(Meirow et al., 2007), and ROS overproduction(Song et al., 2016). Research on organ aging mechanisms can be roughly divided into free radical theory, metabolism theory, telomere theory, and immune system degeneration theory, but none of them can fully explain the phenomenon(Dennis \& Thompson, 2014; Ren \& Zhang, 2019; Rizzo et al., 2014). Aging leads to a decline in vascular dysfunction, and it is undeniable that most importantly, for the reversal of POI ovarian function, the increase in vascularization in ovaries to provide an increase in blood nourishment contributes to the acceleration of the healing procedure and scavenging tissue debris(Liang, Li, \& Chung, 2017).

Currently, stem cell transplantation is generally considered a promising therapeutic for preserving or recovering lost, damaged, or aging tissues. Transplantation of stem cells can promote homing and differentiation in injured ovaries(Tuan \& O'Brien, 2015). Therefore, the present study aimed to analyze the effect of PBMC + PRP transplantation on the vasculature of ovarian tissues in NOA rats and investigated the correlation between angiogenesis of the granulosa on the recovery of ovarian function in NOA rats.

Our previous studies reported that intraovarian injection of PBMCs restores fertility in a chemotherapyinduced POI rat model. However, the efficiency of PBMCs and PRP transplantation in NOA rats has not been explored. Therefore, we planned to evaluate the therapeutic effect and underlying mechanisms of PBMCs or PBMCs + PRP on NOA rats. PBMCs alone or in combination with PRP may be a facile, quick, accessible, safe, and relatively cheap alternative therapeutic strategy to revert NOA-related pathology.

PBMCs maintain the biological properties of other stem cells and possess a great ability to proliferate and differentiate. In addition, compared with other stem cells, PBMCs have many advantages: (1) the process of obtaining PBMCs and PRP creates less pain for patients; (2) most vitally, they can be obtained repeatedly and easily; and (3) autologous transplantation of PBMCs and PRP will not lead to severe immunological rejection. Therefore, PBMCs and PRP may be an ideal replacement for BMSCs, promoting clinical use with promising potential.

Paracrine cytokines exert substantial effects in repairing ovarian function by inhibiting granulosa cell apoptosis(Q. Zhang et al., 2017), promoting angiogenesis(Cho et al., 2021; Seok et al., 2020), and 
reducing oxidative stress( $\mathrm{Na}$ et al., 2020; Seok et al., 2020). As a consequence, stem cells could reconstitute ovarian functions. Many studies have indicated that the reproductive treatment effect of stem cells is linked to their secretome, which is rich in bioactive factors that support ovarian function(Liu et al., 2019). These molecules include insulin-like growth factor (IGF)(Ling et al., 2019), vascular endothelial growth factor (VEGF)(Cho et al., 2021), epidermal growth factor (EGF)(Ding et al., 2020), and other growth factors that trigger cell growth, differentiation, and immunoregulation to restore ovarian function. On the other hand, it is well known that PRP is highly enriched in platelets and growth factors, such as VEGF, TGF- $\beta$, HGF, IGF-1, GDF-9, BMP15, and EGF(Alsousou, Thompson, Harrison, Willett, \& Franklin, 2015; Alves \& Grimalt, 2018; Dehghani, Aboutalebi, Esmaeilpour, Panjehshahin, \& Bordbar, 2018). Moreover, among the many proangiogenic factors, VEGF is one of the major candidates for the regulation of angiogenesis in the ovary(Ferrara, Gerber, \& LeCouter, 2003). Therefore, we further investigated the potential role of VEGF in ovarian vasculature and follicular development after PBMC + PRP treatment and explored the regulatory mechanism. Our data showed that the functional recovery effect of PBMC and PBMC + PRP transplantation on NOA rats is likely to benefit from the upregulation of VEGF secretion.

Growing evidence has shown that energy metabolism disorder of granulosa cells is an important cause of abnormal follicular genesis and fertility decline in females(S. Zhang et al., 2020). It is widely accepted that the function of granulosa cells is essential for the growth and development of mammalian oocytes(Buccione, Schroeder, \& Eppig, 1990). Since oocytes themselves cannot carry out some metabolic processes, such as glycolysis and amino acid uptake, these processes are dependent on the cooperation of granulosa cells(Sugiura \& Eppig, 2005). The effects of glycolysis metabolism during in vivo and in vitro cytoplasmic maturation of oocytes are essential(Boland, Humpherson, Leese, \& Gosden, 1994; H. L. Xie et al., 2016). In particular, pyruvate and lactic acid both play vital roles in follicle formation and oocyte maturation(Tu, Lei, Huo, Le, \& Zhang, 2019). However, oocytes themselves lack glycolysis ability, and the energy of follicle formation is mainly provided by metabolites such as pyruvate and lactic acid produced during glycolysis of granulosa cells(Sugiura, Pendola, \& Eppig, 2005). Quiescent primordial follicles in the ovarian cortex undergo glycolysis/Krebs cycle located away from the vasculature in a hypoxic environment(Makanji, Tagler, Pahnke, Shea, \& Woodruff, 2014). As follicles grow to the secondary stage, they move to the vascular ovarian medulla. From secondary follicles growing to antral follicles, vascularization of the theca cell layer occurs(Brown \& Russell, 2014), providing increased access to oxygen, and oocytes shift toward metabolism consistent with oxidative phosphorylation. Increased oxygen supply promotes oxidative phosphorylation in mural granulosa cells. With the increase in oxygen supply, the glycolysis pathway is upregulated, consistent with previous studies(Harris, Leese, Gosden, \& Picton, 2009). It has long been believed that oocytes are passive recipients of the nutrients produced by cumulus cells, at least with regard to glucose metabolism. Actually, oocytes orchestrate the rate of follicular development and the expression of glycolysis genes in the surrounding granulosa cells(Sugiura \& Eppig, 2005).

Then, we examined the changes in the products and rate-limiting enzymes during glycolysis in granulosa cells. Our data showed that the HK, PKM2, PFK, SIRT1, and LDH-A genes related to glycolysis in 
granulosa cells were upregulated. This result indicated that glycolysis in the ovaries of NOA rats was decreased compared with that in young rats, and PBMC transplantation treatment reversed these processes, suggesting that PBMC transplantation could improve the glycolytic capacity of granulosa cells. Furthermore, combined with PRP injection, there were additive effects. However, the mechanisms by which PBMCs or PBMCs combined with PRP regulate angiogenesis and glycolysis under different conditions have not been fully elucidated. The protective effect verified it as a potential therapeutic method that may be widely used in various pathological conditions.

HRT is widely used in POI patients but has limitations. For example, it increases the risk of heart disease, stroke, breast cancer, and colorectal cancer in POI patients(Sullivan, Sarrel, \& Nelson, 2016). Ovarian tissue cryopreservation is a new therapy employed in POF patients, but the low survival rate in the thawed ovary makes it difficult to conceive naturally after ovarian tissue transplantation (Silber, 2016). Various stem cell transplantation methods have been shown to both effectively restore ovarian function and promote fertility in preclinical animal experiments(Igboeli et al., 2020; Seok et al., 2020). Transplantation of stem cells can reconstitute ovarian functions that are sustained for a limited short period, limiting the potential application of stem cells in the preservation of fertility and endocrine function. The artificial ovary is also a promising alternative that will be used in in vitro growth and maturation approaches or to improve ovarian transplant in the future. Although it has been applied successfully in animal models, its efficacy and safety have to be proven before it is clinically used for patients(Sfakianoudis et al., 2020).

Our study provides new insights into the great clinical potential of PBMCs combined with PRP in treating NOA. PBMCs combined with PRP restored structure and function in ovarian aging and premature aging rat models. PRP greatly extends the effects of follicle development and endocrine functions in PBMCs. Although our study focused on using PRP to extend the functions of PBMC transplants, our findings may also have implications in extending the efficacy of other somatic stem cells in regeneration and transplantation. However, more studies are required to evaluate the feasibility, safety, and efficacy of fertility-preserving methods. Improved therapeutic strategies to prevent natural aging-induced ovarian function decline are needed for these patients.

\section{Conclusions}

Intraovarian transplantation of PBMCs resulted in restoration of the normal estrus cycle, recovery of serum sex hormone levels, increased follicle numbers at all stages, and restoration of fertility. Importantly, PBMCs transplantation into ovaries by local ovarian injection could exert the most conspicuous effect on antagonizing age-associated ovarian hypofunction without any sign of tumorigenicity. Moreover, PRP could enhance these effects, which suggested the potential value of stem cells and PRP in NOA and provided a new strategy for female fertility preservation.

\section{Abbreviations}




\begin{tabular}{|l|}
\hline NOA: Natural ovarian aging \\
\hline POI: Premature ovarian insufficiency \\
\hline POF: Premature ovarian failure \\
\hline PBMCs: Peripheral blood mononuclear cells \\
\hline PRP: Platelet-rich plasma \\
\hline G-CSF: Granulocyte colony-stimulating factor \\
\hline SD: Sprague-Dawley \\
\hline HRT: Hormone replacement therapy \\
\hline CTX: Cyclophosphamide \\
\hline PBS: Phosphate buffered saline \\
\hline IHC: Immunohistochemistry \\
\hline IF: Immunofluorescence \\
\hline E2: Estradiol-17 $\beta$ \\
\hline AMH: Anti-Mullerian hormone \\
\hline FSH: Follicle-stimulating hormone \\
\hline VEGF: Vascular endothelial growth factor \\
\hline CD31: Cluster of differentiation 31 \\
\hline a-SMA: a-smooth muscle actin \\
\hline SIRT1: Sirtuin 1 \\
\hline LDH-A: Lactate dehydrogenase A \\
\hline HK: Hexokinase \\
\hline PFK: Phosphofructokinase \\
\hline PKM2: Pyruvate kinase isozyme M2 \\
\hline SRY: Sex-Related Y Genes \\
\hline
\end{tabular}

\section{Declarations}

\section{Availability of data and materials}

The data that support the findings of this study are available from the corresponding author upon reasonable request. 


\section{Ethics approval and consent to participate}

This research was approved by the Ethics Committee of the Experimental Animal Center of Guangxi Medical University (NO.202003002).

\section{Consent for publication}

Not applicable.

\section{Availability of data and materials}

The datasets used and/or analyzed during the current study are available from the corresponding author on reasonable request.

\section{Competing interests}

The authors do not have any conflicts of interest to declare.

\section{Funding}

This study was supported by grants from the National Natural Science Foundation of China (NO. 81871172), the Natural Science Foundation of Guangxi Zhuang Autonomous Region (NO. 2018GXNSFDA050017 and NO. 2019GXNSFFA245013), the Guangxi Medical University Training Program for Distinguished Young Scholars, and Guangxi Scholarship Fund of Guangxi Education Department of China.

\section{Authors' contributions}

Lifang Yuan: Conceptualization, Methodology, Project Administration, Investigation,Formal Analysis, Validation, Visualization, Writing, and Final Approval, Weiyu Huang: Investigation, Formal Analysis, Writing- Reviewing, Editing, and Final Approval, Yin Bi: Investigation, Formal Analysis, Writing, and Final Approval, Saiqiong Qin: Investigation, Formal Analysis, Writing, and Final Approval, Xi Wang: Investigation, Formal Analysis, Writing, and Final Approval, Ting Li: Validation, Visualization and Final Approval, Peiru Wei: Validation, Visualization and Final Approval,Jiebing Du: Validation, Visualization and Final Approval, Ling Zhao: Validation, Visualization and Final Approval, Bo Liu: Methodology, Project Administration, Supervision, and Final Approval, Yihua Yang: Conceptualization, Funding, Resources, Supervision, Methodology, Project Administration, Investigation, Formal Analysis, Validation, Visualization, Writing, and Final Approval.

\section{Acknowledgments}

The authors acknowledge Mr. Jiaquan Li for assistance with H\&E and IHC staining.

\section{References}


1. Ahmadian, S., Sheshpari, S., Pazhang, M., Bedate, A. M., Beheshti, R., Abbasi, M. M., .. Mahdipour, M. (2020). Intra-ovarian injection of platelet-rich plasma into ovarian tissue promoted rejuvenation in the rat model of premature ovarian insufficiency and restored ovulation rate via angiogenesis modulation. Reprod Biol Endocrinol, 18(1), 78. doi:10.1186/s12958-020-00638-4

2. Ahmed, H. H., Salem, A. M., Atta, H. M., Eskandar, E. F., Farrag, A. R., Ghazy, M. A.,. .. Aglan, H. A. (2016). Updates in the pathophysiological mechanisms of Parkinson's disease: Emerging role of bone marrow mesenchymal stem cells. World J Stem Cells, 8(3), 106-117. doi:10.4252/wjsc.v8.i3.106

3. Alsousou, J., Thompson, M., Harrison, P., Willett, K., \& Franklin, S. (2015). Effect of platelet-rich plasma on healing tissues in acute ruptured Achilles tendon: a human immunohistochemistry study. Lancet, 385 Suppl 1, S19. doi:10.1016/s0140-6736(15)60334-8

4. Alves, R., \& Grimalt, R. (2018). A Review of Platelet-Rich Plasma: History, Biology, Mechanism of Action, and Classification. Skin Appendage Disord, 4(1), 18-24. doi:10.1159/000477353

5. Aydin, O., Karaca, G., Pehlivanli, F., Altunkaya, C., Uzun, H., Özden, H.,. .. Güler, O. (2018). Platelet-Rich Plasma May Offer a New Hope in Suppressed Wound Healing When Compared to Mesenchymal Stem Cells. J Clin Med, 7(6). doi:10.3390/jcm7060143

6. Aziz, N. S., Yusop, N., \& Ahmad, A. (2020). Importance of Stem Cell Migration and Angiogenesis Study for Regenerative Cell-based Therapy: A Review. Curr Stem Cell Res Ther, 15(3), 284-299. doi:10.2174/1574888x15666200127145923

7. Beard, J. R., Officer, A., de Carvalho, I. A., Sadana, R., Pot, A. M., Michel, J. P., .. Chatterji, S. (2016). The World report on ageing and health: a policy framework for healthy ageing. Lancet, 387(10033), 2145-2154. doi:10.1016/s0140-6736(15)00516-4

8. Boland, N. I., Humpherson, P. G., Leese, H. J., \& Gosden, R. G. (1994). Characterization of follicular energy metabolism. Hum Reprod, 9(4), 604-609. doi:10.1093/oxfordjournals. humrep.a138557

9. Broekmans, F. J., Soules, M. R., \& Fauser, B. C. (2009). Ovarian aging: mechanisms and clinical consequences. Endocr Rev, 30(5), 465-493. doi:10.1210/er.2009-0006

10. Brown, H. M., \& Russell, D. L. (2014). Blood and lymphatic vasculature in the ovary: development, function and disease. Hum Reprod Update, 20(1), 29-39. doi:10.1093/humupd/dmt049

11. Buccione, R., Schroeder, A. C., \& Eppig, J. J. (1990). Interactions between somatic cells and germ cells throughout mammalian oogenesis. Biol Reprod, 43(4), 543-547. doi:10.1095/biolreprod43.4.543

12. Cho, J., Kim, T. H., Seok, J., Jun, J. H., Park, H., Kweon, M., .. Kim, G. J. (2021). Vascular remodeling by placenta-derived mesenchymal stem cells restores ovarian function in ovariectomized rat model via the VEGF pathway. Lab Invest, 101(3), 304-317. doi:10.1038/s41374-020-00513-1

13. Dadashzadeh, A., Moghassemi, S., Shavandi, A., \& Amorim, C. A. (2021). A review on biomaterials for ovarian tissue engineering. Acta Biomater. doi:10.1016/j.actbio.2021.08.026

14. Dehghani, F., Aboutalebi, H., Esmaeilpour, T., Panjehshahin, M. R., \& Bordbar, H. (2018). Effect of platelet-rich plasma (PRP) on ovarian structures in cyclophosphamide-induced ovarian failure in 
female rats: a stereological study. Toxicol Mech Methods, 28(9), 653-659.

doi:10.1080/15376516.2018.1491662

15. Dennis, E. L., \& Thompson, P. M. (2014). Functional brain connectivity using fMRI in aging and Alzheimer's disease. Neuropsychol Rev, 24(1), 49-62. doi:10.1007/s11065-014-9249-6

16. Ding, C., Zou, Q., Wu, Y., Lu, J., Qian, C., Li, H., \& Huang, B. (2020). EGF released from human placental mesenchymal stem cells improves premature ovarian insufficiency via NRF2/HO-1 activation. Aging (Albany NY), 12(3), 2992-3009. doi:10.18632/aging.102794

17. El Andaloussi, A., Igboeli, P., Amer, A., \& Al-Hendy, A. (2018). Intravenous Infusion of Nucleated Peripheral Blood Cells Restores Fertility in Mice with Chemotherapy-Induced Premature Ovarian Failure. Biomedicines, 6(3). doi:10.3390/biomedicines6030093

18. Ferrara, N., Gerber, H. P., \& LeCouter, J. (2003). The biology of VEGF and its receptors. Nat Med, 9(6), 669-676. doi:10.1038/nm0603-669

19. Fontana, J., Martínková, S., Petr, J., Žalmanová, T., \& Trnka, J. (2020). Metabolic cooperation in the ovarian follicle. Physiol Res, 69(1), 33-48. doi:10.33549/physiolres.934233

20. Grosbois, J., Devos, M., \& Demeestere, I. (2020). Implications of Nonphysiological Ovarian Primordial Follicle Activation for Fertility Preservation. Endocr Rev, 41(6). doi:10.1210/endrev/bnaa020

21. Harris, S. E., Leese, H. J., Gosden, R. G., \& Picton, H. M. (2009). Pyruvate and oxygen consumption throughout the growth and development of murine oocytes. Mol Reprod Dev, 76(3), 231-238. doi:10.1002/mrd.20945

22. Huang, Q., Liu, B., Jiang, R., Liao, S., Wei, Z., Bi, Y.,. .. Qin, A. (2019). G-CSF-mobilized peripheral blood mononuclear cells combined with platelet-rich plasma accelerate restoration of ovarian function in cyclophosphamide-induced POI ratst. Biol Reprod, 101(1), 91-101. doi:10.1093/biolre/ioz077

23. Igboeli, P., El Andaloussi, A., Sheikh, U., Takala, H., ElSharoud, A., McHugh, A.,. .. Al-Hendy, A. (2020). Intraovarian injection of autologous human mesenchymal stem cells increases estrogen production and reduces menopausal symptoms in women with premature ovarian failure: two case reports and a review of the literature. J Med Case Rep, 14(1), 108. doi:10.1186/s13256-020-02426-5

24. Kalich-Philosoph, L., Roness, H., Carmely, A., Fishel-Bartal, M., Ligumsky, H., Paglin, S.,. .. Meirow, D. (2013). Cyclophosphamide triggers follicle activation and "burnout"; AS101 prevents follicle loss and preserves fertility. Sci Trans/ Med, 5(185), 185ra162. doi:10.1126/scitranslmed.3005402

25. Kang, H. J., Kim, M. K., Lee, H. Y., Park, K. W., Lee, W., Cho, Y. S., .. Kim, H. S. (2012). Five-year results of intracoronary infusion of the mobilized peripheral blood stem cells by granulocyte colonystimulating factor in patients with myocardial infarction. Eur Heart J, 33(24), 3062-3069. doi:10.1093/eurheartj/ehs231

26. Khaleghi, S., Fathi, R., Eivazkhani, F., Moini, A., Novin, M. G., Ebrahimi, B., \& Nazarian, H. (2021). TwoDecade Experience of Royan Institute in Obtaining Mature Oocyte from Cryopreserved Ovarian Tissue: In Vitro and In Vivo Approaches. Reprod Sci. doi:10.1007/s43032-021-00728-7

27. Koh, H., Rah, W. J., Kim, Y. J., Moon, J. H., Kim, M. J., \& Lee, Y. H. (2018). Serial Changes of Cytokines in Children with Cerebral Palsy Who Received Intravenous Granulocyte-colony Stimulating Factor 
Followed by Autologous Mobilized Peripheral Blood Mononuclear Cells. J Korean Med Sci, 33(21), e102. doi:10.3346/jkms.2018.33.e102

28. Liang, N., Li, Y., \& Chung, H. Y. (2017). Two natural eudesmane-type sesquiterpenes from Laggera alata inhibit angiogenesis and suppress breast cancer cell migration through VEGF- and Angiopoietin 2-mediated signaling pathways. Int J Oncol, 51(1), 213-222. doi:10.3892/ijo.2017.4004

29. Ling, L., Feng, X., Wei, T., Wang, Y., Wang, Y., Wang, Z.,. .. Xiong, Z. (2019). Human amnion-derived mesenchymal stem cell (hAD-MSC) transplantation improves ovarian function in rats with premature ovarian insufficiency (POI) at least partly through a paracrine mechanism. Stem Cell Res Ther, 10(1), 46. doi:10.1186/s13287-019-1136-x

30. Liu, R., Zhang, X., Fan, Z., Wang, Y., Yao, G., Wan, X.,. .. Yu, L. (2019). Human amniotic mesenchymal stem cells improve the follicular microenvironment to recover ovarian function in premature ovarian failure mice. Stem Cell Res Ther, 10(1), 299. doi:10.1186/s13287-019-1315-9

31. Makanji, Y., Tagler, D., Pahnke, J., Shea, L. D., \& Woodruff, T. K. (2014). Hypoxia-mediated carbohydrate metabolism and transport promote early-stage murine follicle growth and survival. Am J Physiol Endocrinol Metab, 306(8), E893-903. doi:10.1152/ajpendo.00484.2013

32. Meirow, D., Dor, J., Kaufman, B., Shrim, A., Rabinovici, J., Schiff, E.,. .. Fridman, E. (2007). Cortical fibrosis and blood-vessels damage in human ovaries exposed to chemotherapy. Potential mechanisms of ovarian injury. Hum Reprod, 22(6), 1626-1633. doi:10.1093/humrep/dem027

33. Na, J., Song, J., Kim, H. H., Seok, J., Kim, J. Y., Jun, J. H., \& Kim, G. J. (2020). Human placenta-derived mesenchymal stem cells trigger repair system in TAA-injured rat model via antioxidant effect. Aging (Albany NY), 13(1), 61-76. doi:10.18632/aging.202348

34. Ren, J., \& Zhang, Y. (2018). Targeting Autophagy in Aging and Aging-Related Cardiovascular Diseases. Trends Pharmacol Sci, 39(12), 1064-1076. doi:10.1016/j.tips.2018.10.005

35. Ren, J., \& Zhang, Y. (2019). Genetics and Epigenetics in Aging and Longevity: Myths and Truths. Biochim Biophys Acta Mol Basis Dis, 1865(7), 1715-1717. doi:10.1016/j.bbadis.2019.02.005

36. Rizzo, L. B., Costa, L. G., Mansur, R. B., Swardfager, W., Belangero, S. I., Grassi-Oliveira, R.,. .. Brietzke, E. (2014). The theory of bipolar disorder as an illness of accelerated aging: implications for clinical care and research. Neurosci Biobehav Rev, 42, 157-169. doi:10.1016/j.neubiorev.2014.02.004

37. Sassoli, C., Vallone, L., Tani, A., Chellini, F., Nosi, D., \& Zecchi-Orlandini, S. (2018). Combined use of bone marrow-derived mesenchymal stromal cells (BM-MSCs) and platelet rich plasma (PRP) stimulates proliferation and differentiation of myoblasts in vitro: new therapeutic perspectives for skeletal muscle repair/regeneration. Cell Tissue Res, 372(3), 549-570. doi:10.1007/s00441-0182792-3

38. Seok, J., Park, H., Choi, J. H., Lim, J. Y., Kim, K. G., \& Kim, G. J. (2020). Placenta-Derived Mesenchymal Stem Cells Restore the Ovary Function in an Ovariectomized Rat Model via an Antioxidant Effect. Antioxidants (Basel), 9(7). doi:10.3390/antiox9070591

39. Sfakianoudis, K., Rapani, A., Grigoriadis, S., Retsina, D., Maziotis, E., Tsioulou, P., .. Simopoulou, M. (2020). Novel Approaches in Addressing Ovarian Insufficiency in 2019: Are We There Yet? Cell 
Transplant, 29, 963689720926154. doi:10.1177/0963689720926154

40. Silber, S. (2016). Ovarian tissue cryopreservation and transplantation: scientific implications. $J$ Assist Reprod Genet, 33(12), 1595-1603. doi:10.1007/s10815-016-0814-1

41. Song, C., Peng, W., Yin, S., Zhao, J., Fu, B., Zhang, J., .. Zhang, Y. (2016). Melatonin improves ageinduced fertility decline and attenuates ovarian mitochondrial oxidative stress in mice. Sci Rep, 6, 35165. doi:10.1038/srep35165

42. Spears, N., Lopes, F., Stefansdottir, A., Rossi, V., De Felici, M., Anderson, R. A., \& Klinger, F. G. (2019). Ovarian damage from chemotherapy and current approaches to its protection. Hum Reprod Update, 25(6), 673-693. doi:10.1093/humupd/dmz027

43. Sugiura, K., \& Eppig, J. J. (2005). Society for Reproductive Biology Founders' Lecture 2005. Control of metabolic cooperativity between oocytes and their companion granulosa cells by mouse oocytes. Reprod Fertil Dev, 17(7), 667-674. doi:10.1071/rd05071

44. Sugiura, K., Pendola, F. L., \& Eppig, J. J. (2005). Oocyte control of metabolic cooperativity between oocytes and companion granulosa cells: energy metabolism. Dev Biol, 279(1), 20-30. doi:10.1016/j.ydbio.2004.11.027

45. Sullivan, S. D., Sarrel, P. M., \& Nelson, L. M. (2016). Hormone replacement therapy in young women with primary ovarian insufficiency and early menopause. Fertil Steril, 106(7), 1588-1599. doi:10.1016/j.fertnstert.2016.09.046

46. Tatone, C., Amicarelli, F., Carbone, M. C., Monteleone, P., Caserta, D., Marci, R.,. .. Focarelli, R. (2008). Cellular and molecular aspects of ovarian follicle ageing. Hum Reprod Update, 14(2), 131-142. doi:10.1093/humupd/dmm048

47. Tu, H. Y., Lei, X. C., Huo, P., Le, J. H., \& Zhang, S. (2019). [Energy Demand and Its Regulatory Mechanism during Folliculogenesis]. Zhongguo Yi Xue Ke Xue Yuan Xue Bao, 41(3), 408-414. doi:10.3881/j.issn.1000-503X.10774

48. Tuan, R. S., \& O'Brien, T. (2015). Expression of Concern: Human amniotic epithelial cells can differentiate into granulosa cells and restore folliculogenesis in a mouse model of chemotherapyinduced premature ovarian failure. Stem Cell Res Ther, 6, 240. doi:10.1186/s13287-015-0234-7

49. Wan Jamaludin, W. F., Mohamad Yusoff, F., Ismail, N. A., Mohd Idris, M. R., Palaniappan, S., Ng, C. K. K.,. .. Abdul Wahid, S. F. S. (2018). Autologous mononuclear cells from different sources are seen to improve wound healing in patients with haematological malignancies. Malays J Pathol, 4O(1), 6167.

50. Wang, L., Mei, Q., Xie, Q., Li, H., Su, P., Zhang, L.,. .. Xiang, W. (2021). A comparative study of Mesenchymal Stem Cells transplantation approach to antagonize age-associated ovarian hypofunction with consideration of safety and efficiency. Journal of Advanced Research. doi:10.1016/j.jare.2021.09.001

51. White, Y. A., Woods, D. C., Takai, Y., Ishihara, O., Seki, H., \& Tilly, J. L. (2012). Oocyte formation by mitotically active germ cells purified from ovaries of reproductive-age women. Nat Med, 18(3), 413421. doi: $10.1038 / \mathrm{nm} .2669$ 
52. Xie, H. L., Wang, Y. B., Jiao, G. Z., Kong, D. L., Li, Q., Li, H., .. Tan, J. H. (2016). Effects of glucose metabolism during in vitro maturation on cytoplasmic maturation of mouse oocytes. Sci Rep, 6 , 20764. doi:10.1038/srep20764

53. Xie, Q., Cheng, Z., Chen, X., Lobe, C. G., \& Liu, J. (2017). The role of Notch signalling in ovarian angiogenesis. J Ovarian Res, 1O(1), 13. doi:10.1186/s13048-017-0308-5

54. Zhang, Q., Bu, S., Sun, J., Xu, M., Yao, X., He, K., \& Lai, D. (2017). Paracrine effects of human amniotic epithelial cells protect against chemotherapy-induced ovarian damage. Stem Cell Res Ther, 8(1), 270. doi:10.1186/s13287-017-0721-0

55. Zhang, S., Tu, H., Yao, J., Le, J., Jiang, Z., Tang, Q.,. .. Lei, X. (2020). Combined use of Diane-35 and metformin improves the ovulation in the PCOS rat model possibly via regulating glycolysis pathway. Reprod Biol Endocrinol, 18(1), 58. doi:10.1186/s12958-020-00613-z

\section{Figures}

(A)

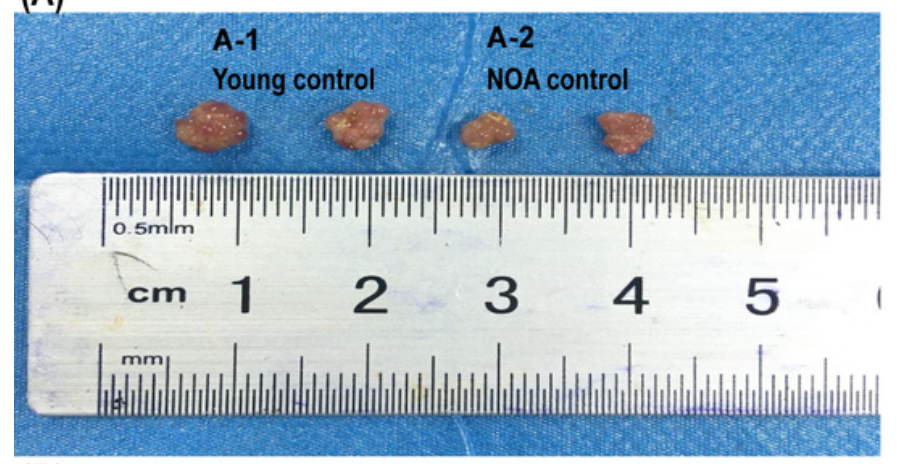

(B)

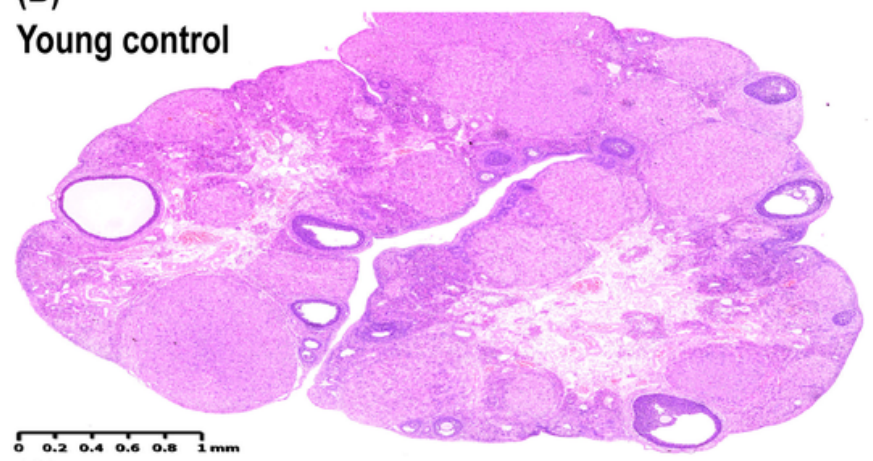

(C)

\section{NOA control}

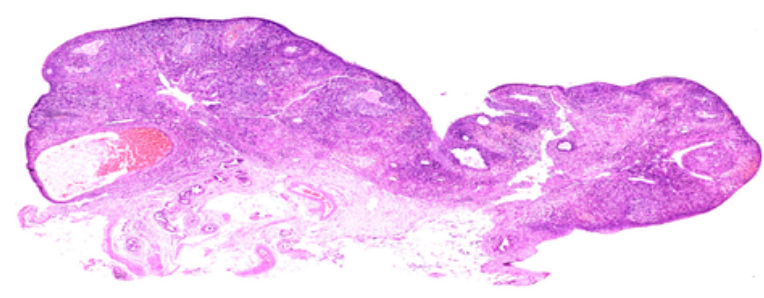

o.2 0.t o.s o.t $1 \mathrm{~mm}$
(D)

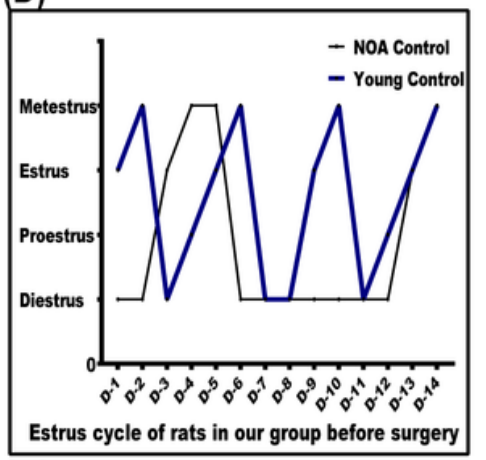

(F)

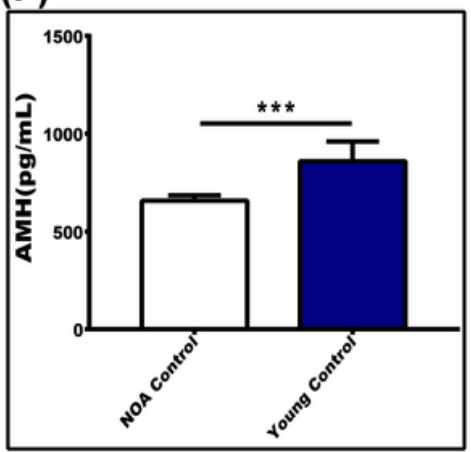

(H)

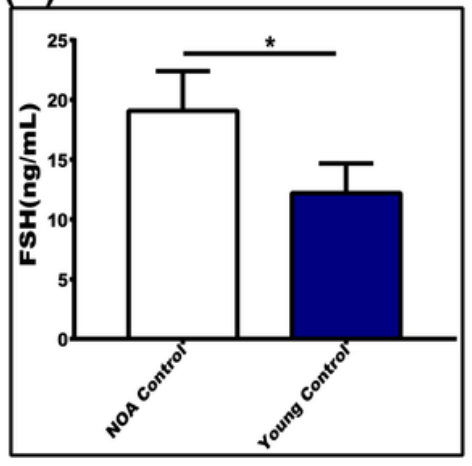

(E)

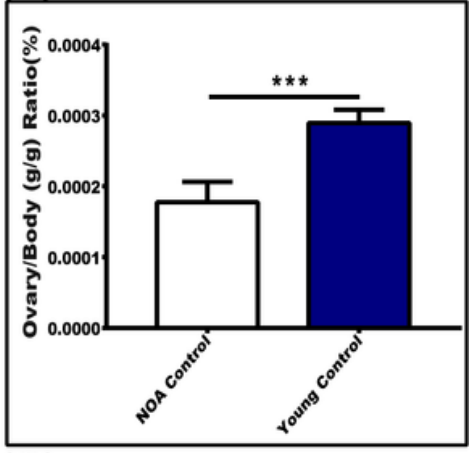

(G)

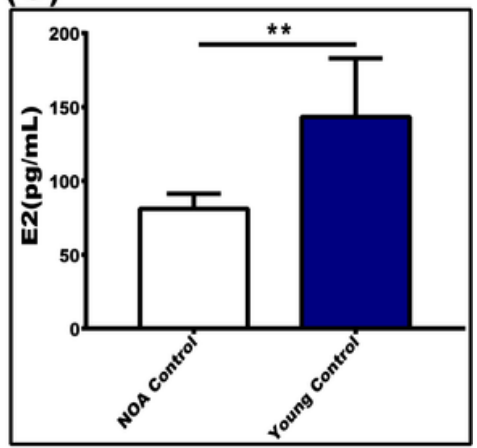

(I)

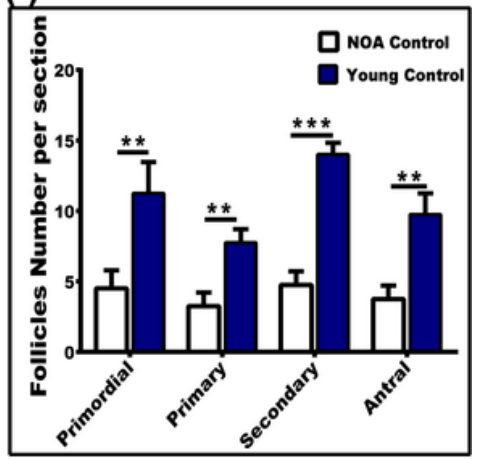

Figure 1 
(A)

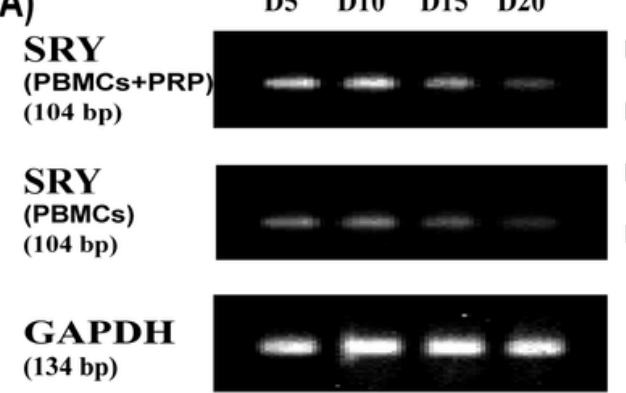

(D)

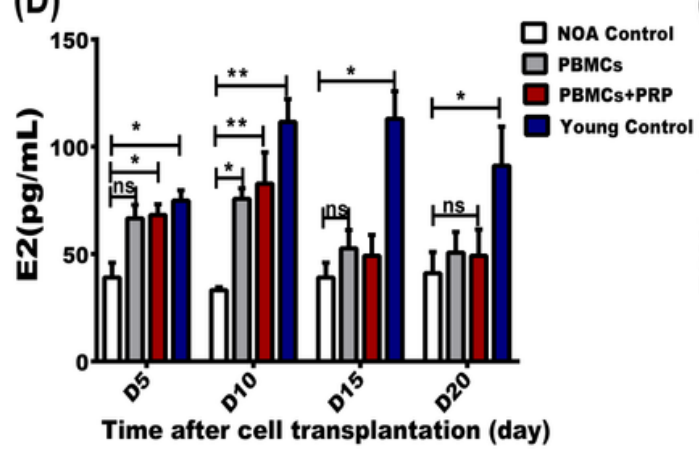

(G)

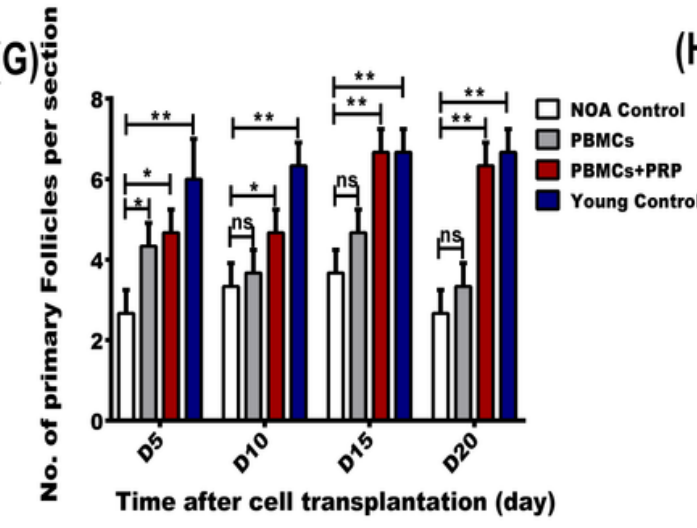

(B)

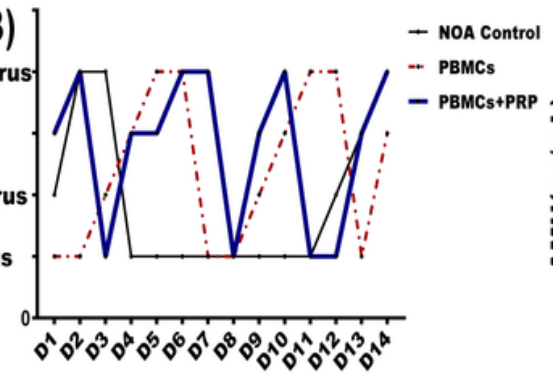

Estrus cycle of rats in our group after surgery

(E)

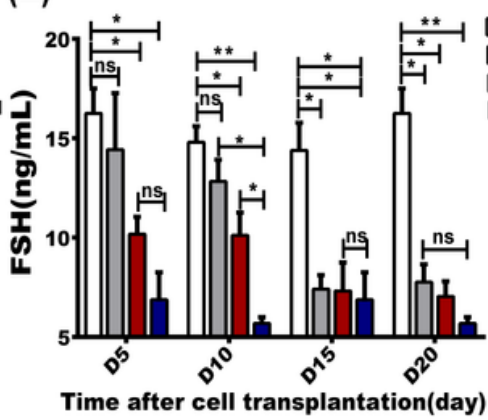

$(\mathbf{F})$

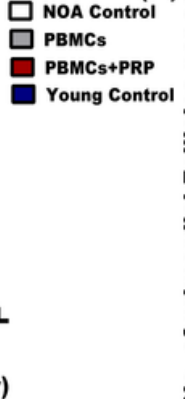

(H) $\frac{5}{\stackrel{0}{0}}$

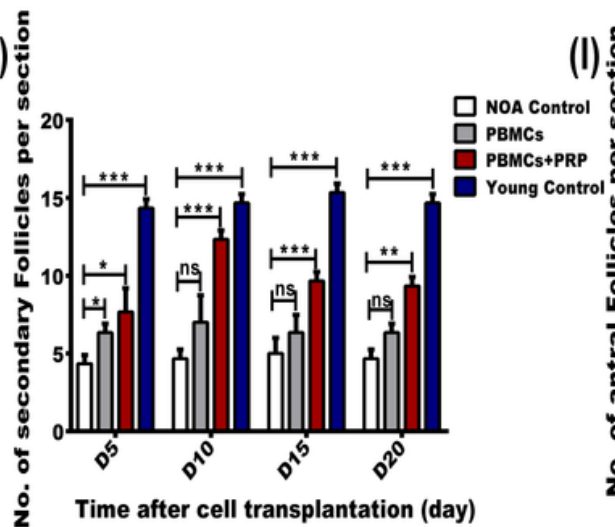

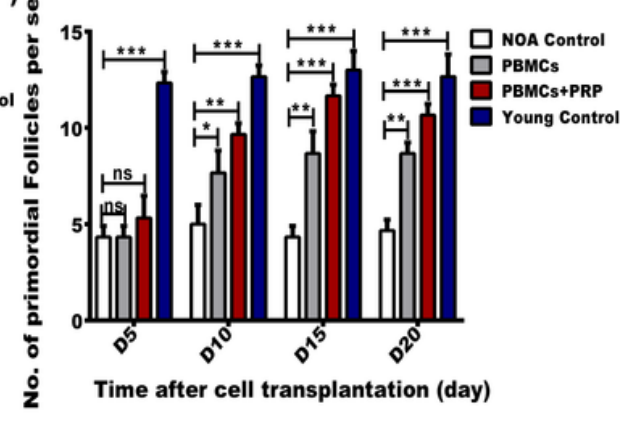

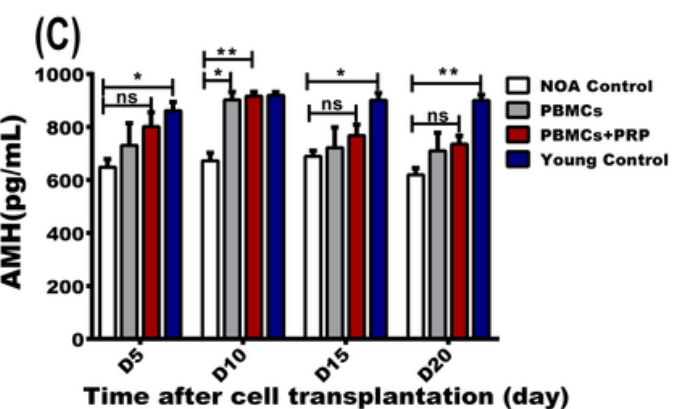

(F)

1)

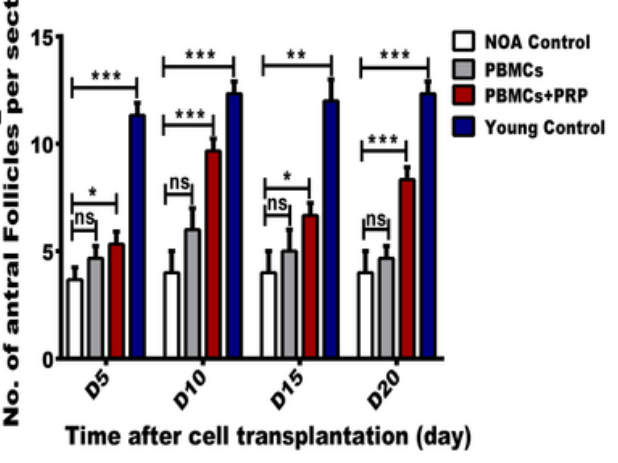

Figure 2

Observation of the curative effect of NOA rat ovarian PBMCs cells transplantation 
NOA Control
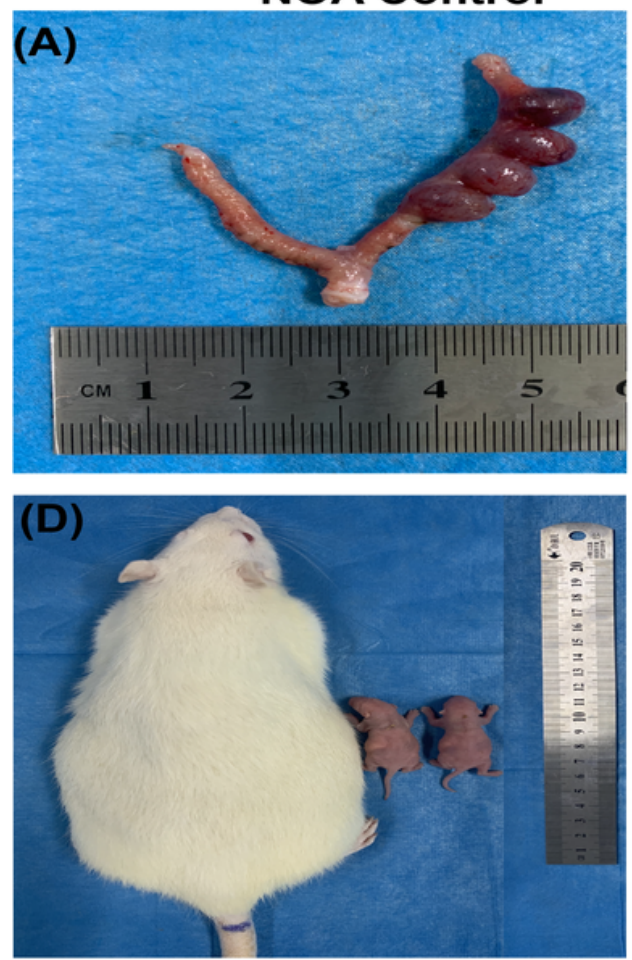

(G)

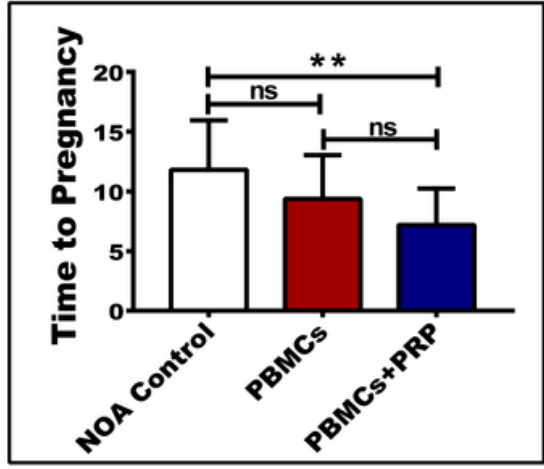

PBMCs
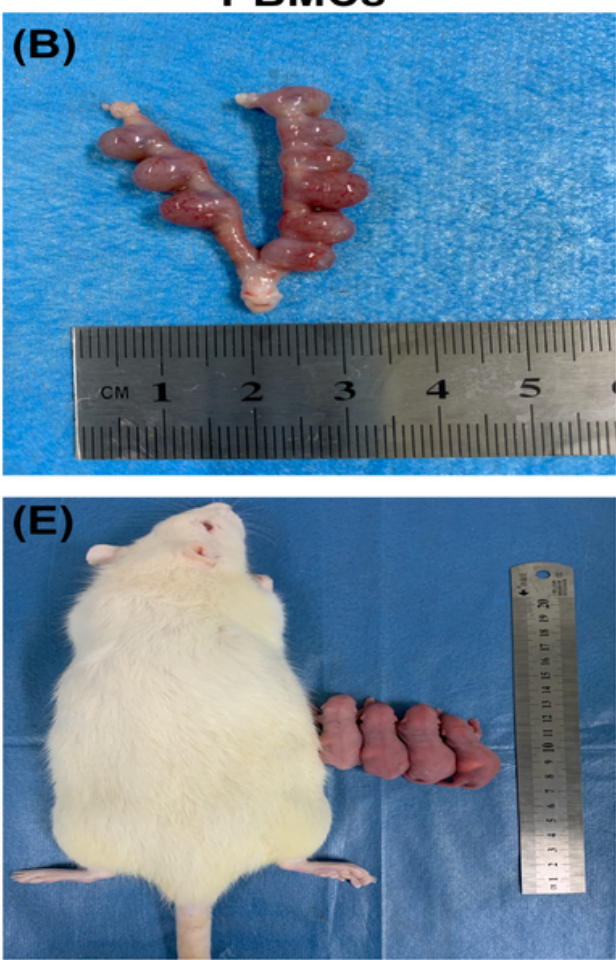

(H)

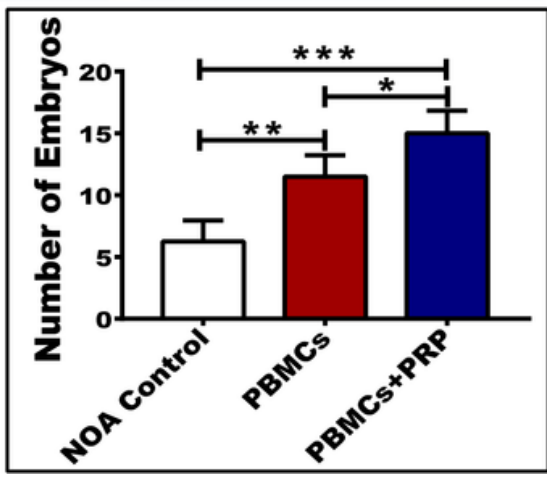

PBMCs+PRP
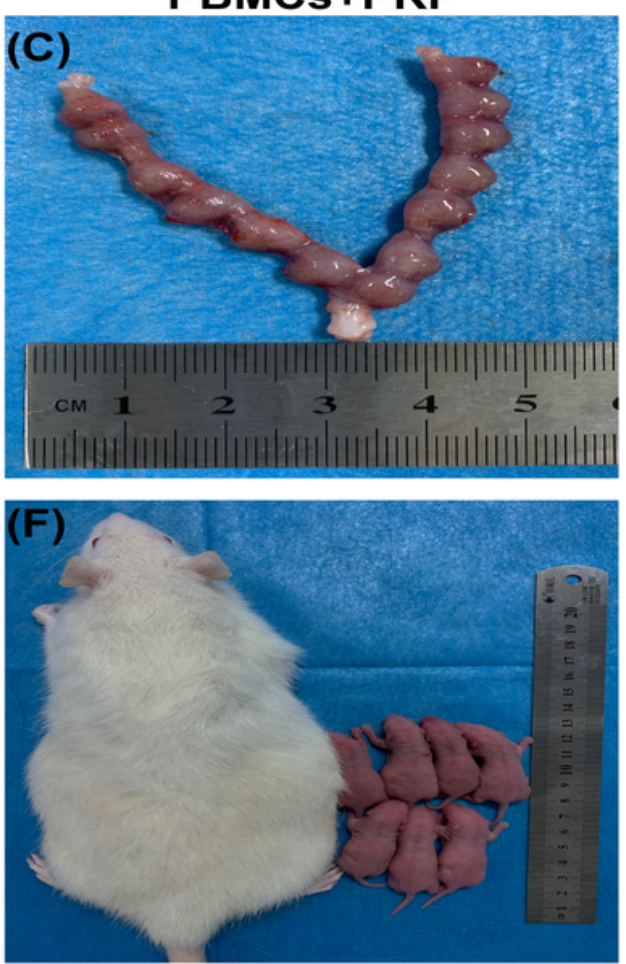

(I)

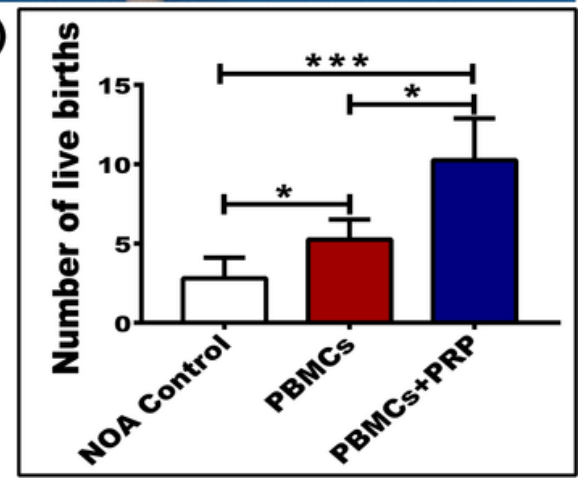

Figure 3

Rescue of embryonic development and live birth by PBMCs or combined with PRP. 

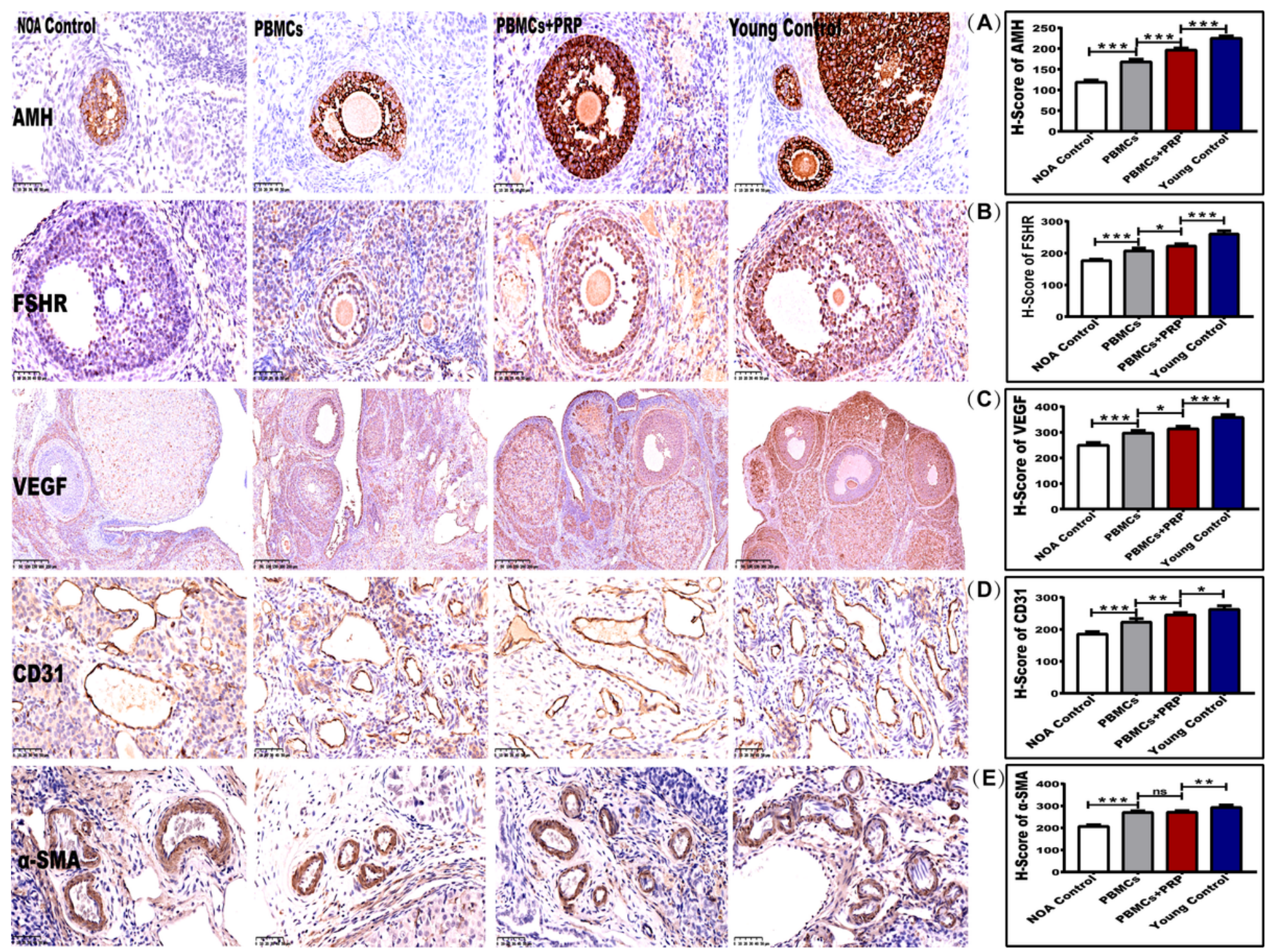

(B)

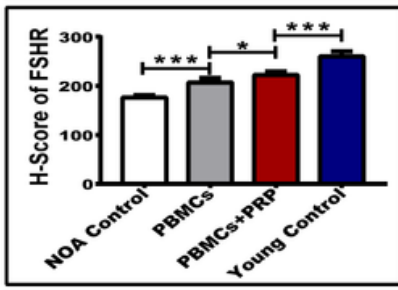

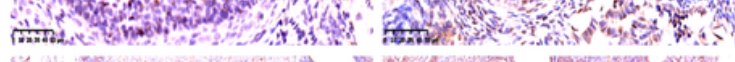
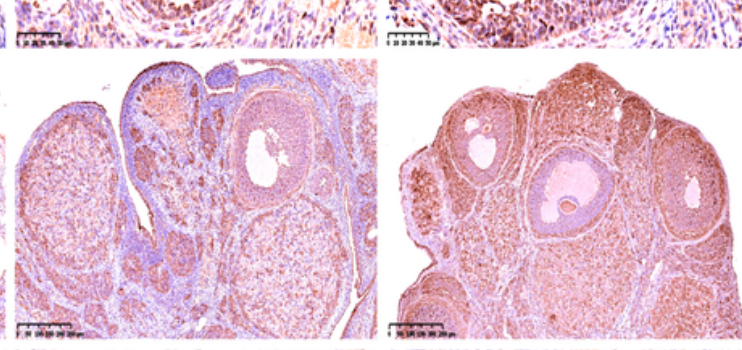

(C)
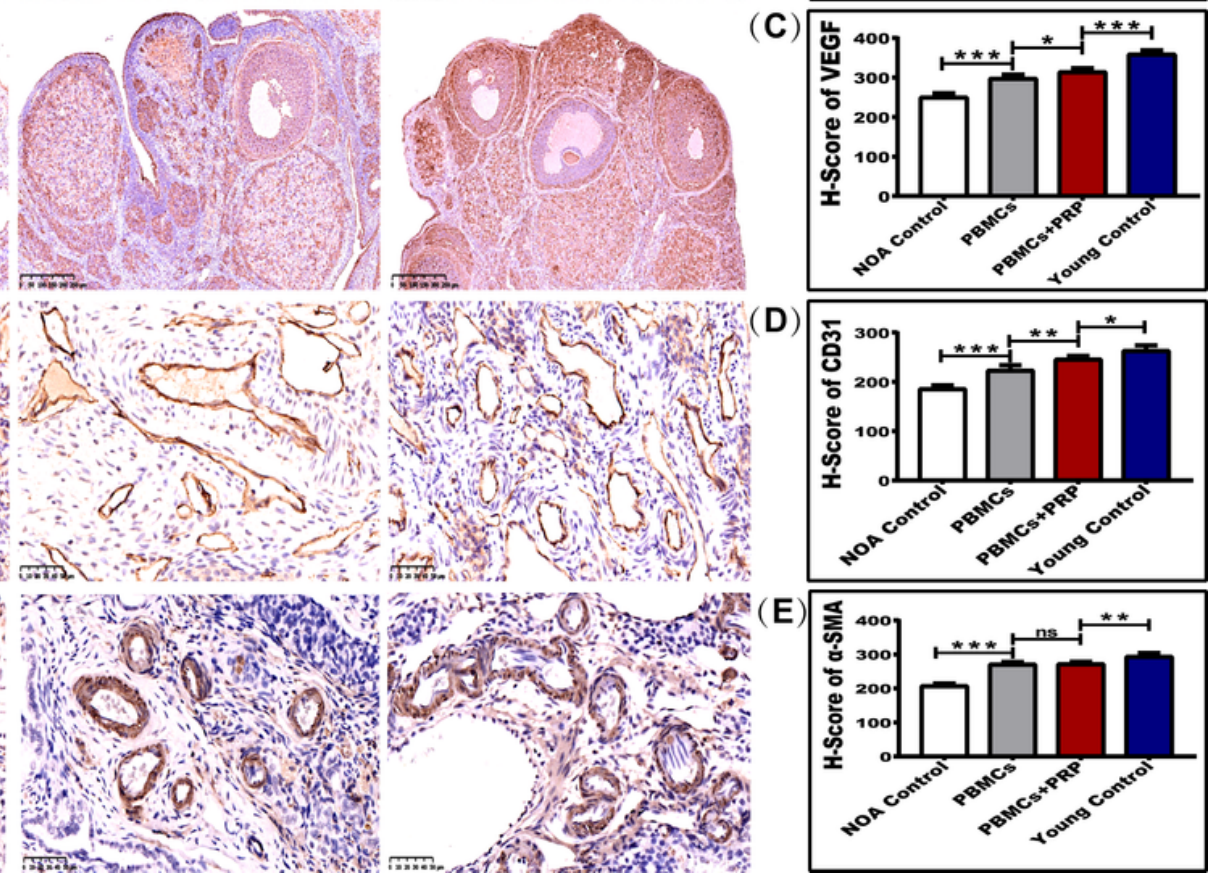

Figure 4

PBMCs and PBMCs+PRP significantly increase the expression of angiogenesis and folliculogenesisrelated markers 

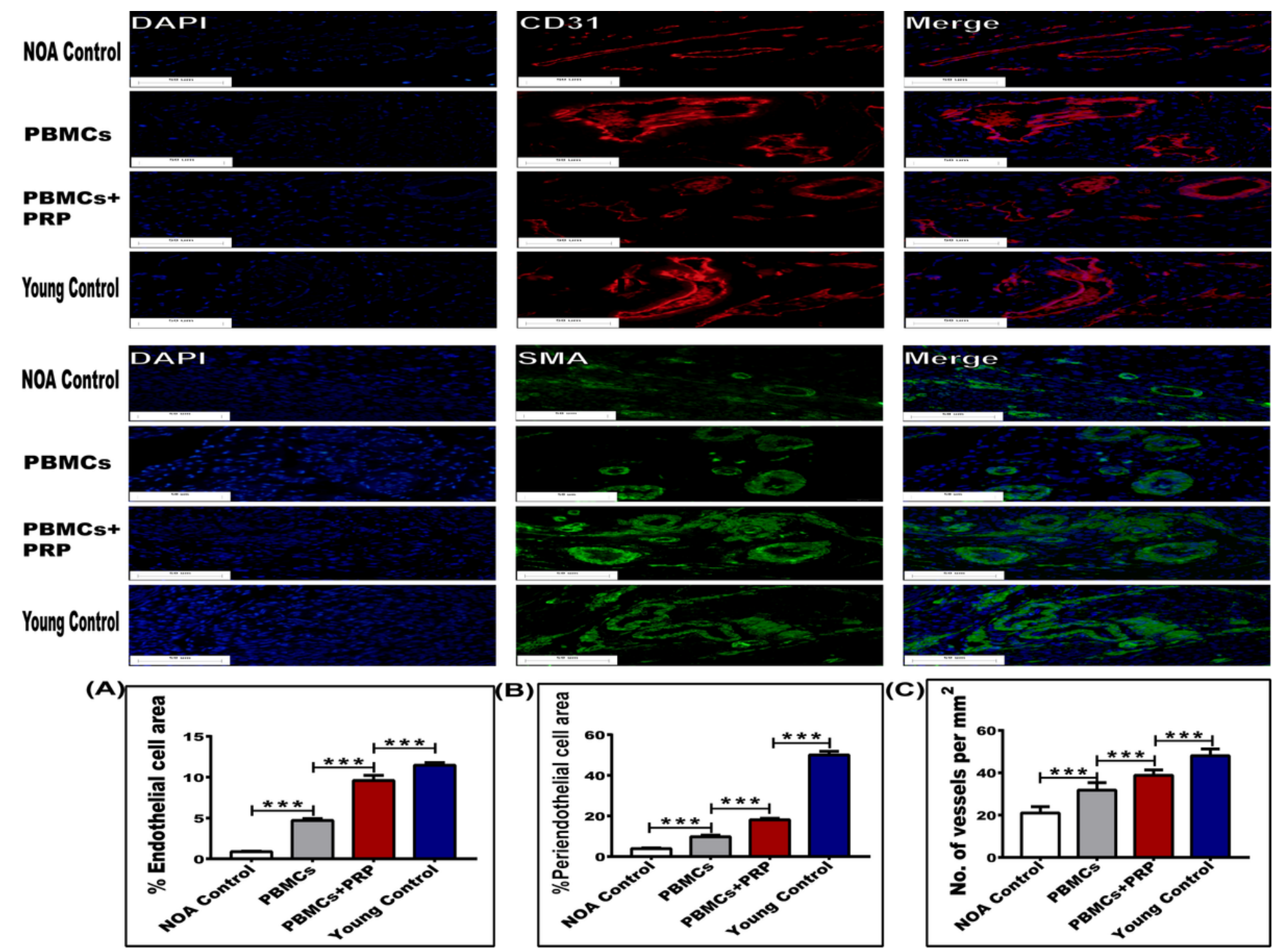

Figure 5

PBMCs and PBMCs+PRP could restore the ovarian function of aged rats by up-regular the ovarian angiogenesis in NOA rats 


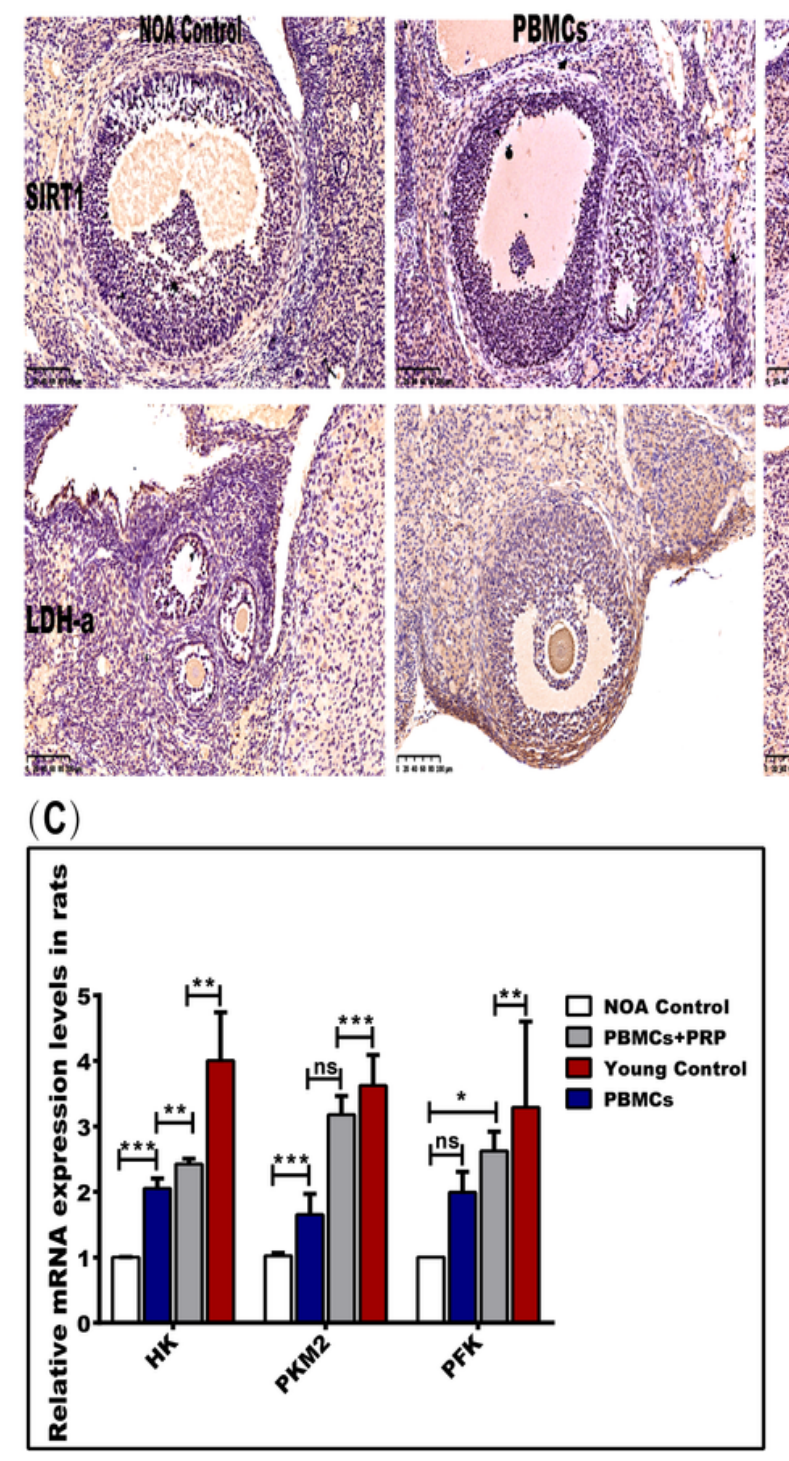

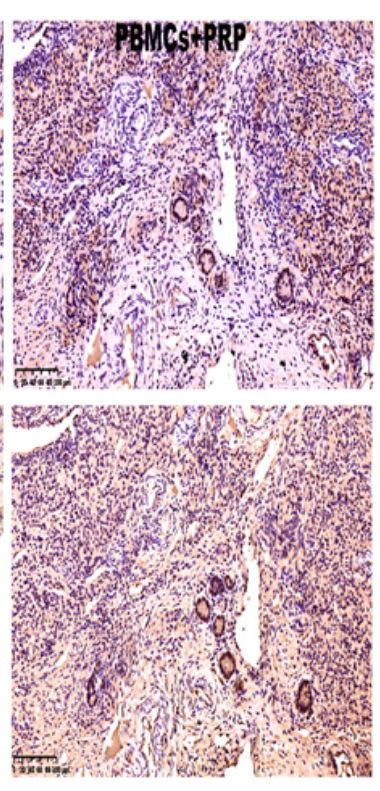

(D)

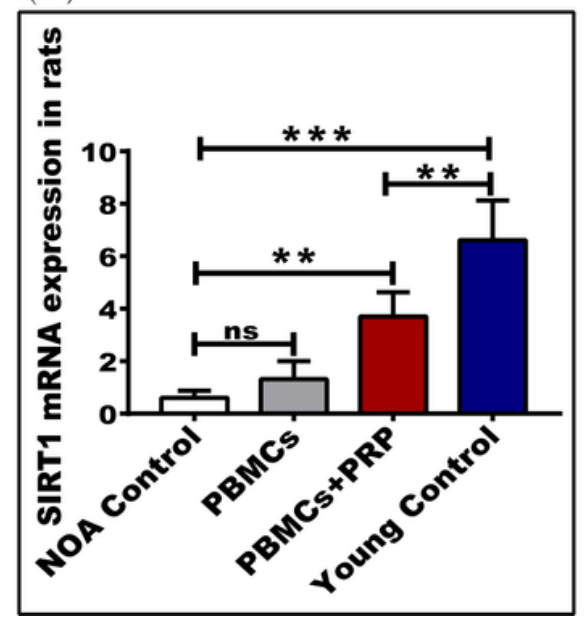

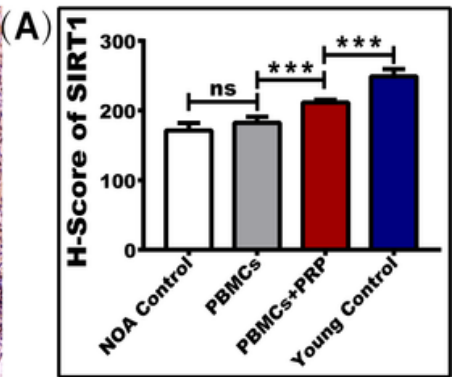

(B)

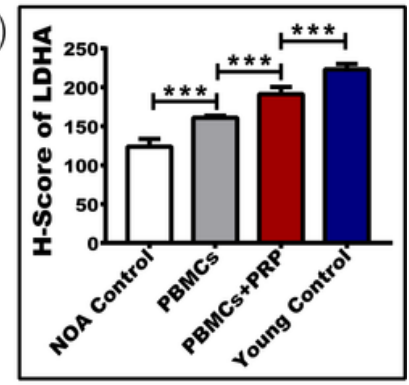

(E)

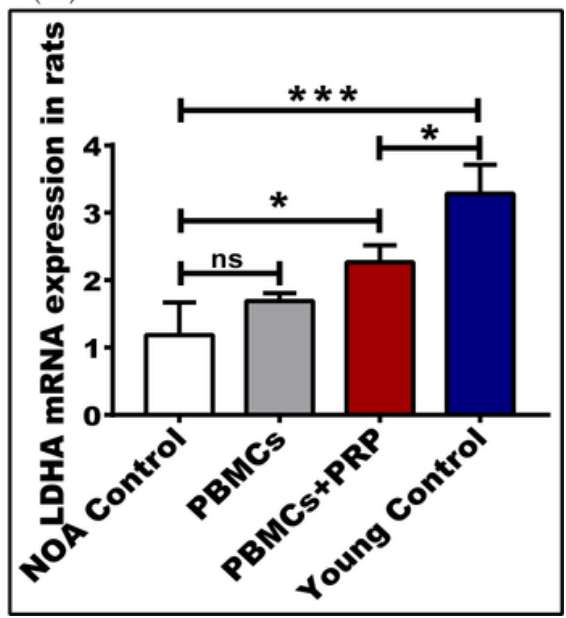

Figure 6

PBMCs restored the ovarian function of aged rats via glycolysis modulation; combined with PRP could help this effect

\section{Supplementary Files}

This is a list of supplementary files associated with this preprint. Click to download.

- Figs1.tif

- Figs2.tif 